\title{
RAPID ULTRAVIOLET SPECTRAL VARIATIONS IN HD 50896 (WN5+?)
}

\author{
A $\mathrm{J}$ Willis and I D Howarth \\ Department of Physics \& Astronomy, UCL, London, UK \\ P S Conti and C D Garmany \\ JILA, University of Colorado, Boulder, Colorado, USA
}

HD 50896 has become one of the most promising candidate WR+collapsar systems ( Moffat 1982 ) exhibiting optical light, RV and polarisation variations with a 3d.763 period ( cf Firmani et al. 1980 ) yet direct evidence for its postulated n.s. component is lacking. UV spectra of analogous $O B X$-ray binaries show P-Cygni profile changes as a function of binary phase which are well understood in terms of anisotropic wind ionisation caused by X-ray emission from the accreting n.s. - the Hatchett \& McCray (1976) effect. Observations of such UV variability in $W R+C$ candidates with a clear phase dependence would thus provide good evidence for any n.s. companion. To this end we have obtained IUE spectra of HD 50896 in sequential NASA/VILSPA shifts over 6 consecutive days in 1983, together with complementary IUE data secured at various epochs during 1978-82, to search for the Hatchett \& McCray effect in this star.

The IUE spectra of HD 50896 do show significant variations in the P-Cygni profiles of NV 1240, CIV 1550, HeII 1640 and, particularly, in NIV 1718 - the line we concentrate on herein. Fig 1 plots the equivalent width $\left(\mathrm{W}_{\mathrm{a}}\right.$ ) of the NIV 1718 P-Cygni absorption component measured in each spectrum against binary phase. Although substantial variability is apparent there is no phase dependence analogous to that observed in aII $O B X$-ray binaries studied with IUE. Thus our data do not provide any supportive evidence for the proposed n.s. companion. Rather, the 1983 data show evidence for rapid variability in the P-Cygni absorption ( in some cases $\sim 30 \%$ in a few hours ) occuring at all phases. This strongly suggests that the observed changes are linked to mechanisms intrinsic to the WN star itself rather than binarity effects. Typically the profile changes are confined to a restricted range of -ve velocities $(-1000$ to $-1800 \mathrm{~km} / \mathrm{s}$ for NIV $)$, considerably less than the wind terminal velocity of about $3000 \mathrm{~km} / \mathrm{s}$. This indicates that variations occur in the physical properties of the accelerating part of the wind. Corresponding profile changes in other lines show extensions to different velocities ( $-2100 \mathrm{~km} / \mathrm{s}$ for HeII; $-2500 \mathrm{~km} / \mathrm{s}$ for NV ) probably reflective of wind stratification in the different ions. Spectra secured during 1978-82 show similar changes in NIV 1718 ( cf Fig 1 ) with evidence for secular effects - some of the 1980 data show markedly stronger values of $\mathrm{W}_{\mathrm{a}}$ 
Fig 1: Measured values of $W_{a}$ ( see text ) for NIV 1718 plotted against binary phase for: (a) IUE data secured in Sept. 1983

(b) 1980 data obtained over consecutive days $\square$ and (c) IUE data obtained at sporadic epochs during 1978-82

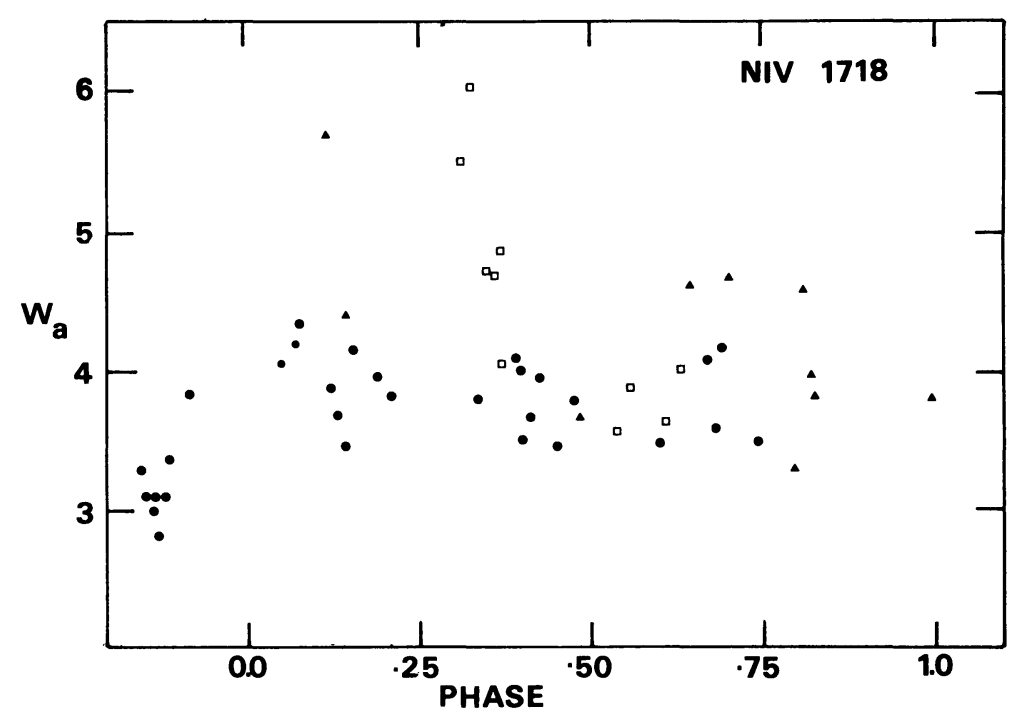

than seen at other times, accompanied by an extension of the P-Cygni absorption edges to higher displaced velocities ( making a unique measurement of the terminal velocity for HD 50896 ambiguous ).

In our view, current wind models are not capable of explaining the observed P-Cygni profile variations in HD 50896. The time scale of the variability ( which can be $\leqslant 3$ hours; we have yet to ascertain any periodicity, and the clear indication that we are dealing with changes to the accelerating region of the wind (close to the WN star ) leads us to conjecture that they may arise from some kind of sub-wind activity in the WR component itself. Recently Vreux ( 1984, preprints ) has found evidence that the optical variability shown by proposed $W R+C$ candidates may more likely result from single star non-radial pulsations rather than spirally-in n.s. scenarios. Perhaps the UV variability of HD 50896 is also explicable in terms of such pulsational activity.

Hatchett,S.P., McCray,R., 1976, ApJ., 211, 552

Firmani,C., et al., 1980, ApJ., 239, $6 \overline{04}$

Moffat,A., 1982, in Proc. IAU Symp. No.99 ( eds. de Loore \& Willis ), 263 